\title{
Calidad del agua subterránea para el riego en el Oasis de Pica, norte de Chile
}

\author{
Groundwater quality for irrigation in Oasis de Pica, north of Chile \\ Venecia Herrera Apablaza ${ }^{1 *}$, Natalia Gutiérrez Roa ${ }^{4}$, Salomé Córdova Molina ${ }^{4}$, José Luque Marín ${ }^{2,4}$, \\ María Idelfonso Carpanchay ${ }^{4}$, Alejandro Flores Riveras ${ }^{4}$, Leonardo Romero A ${ }^{3,4}$
}

\begin{abstract}
RESUMEN
Agricultores de las localidades del Oasis de Pica, precordillera del norte de Chile, aprovechan las aguas subterráneas a distintas profundidades en el riego de cultivos cítricos, frutos tropicales y verduras. Con el objeto de establecer una red de monitoreo y entregar recomendaciones a los regantes, se caracterizaron las propiedades fisicoquímicas de estas aguas, su comportamiento temporal anual y se determinó su calidad para riego, además de interpretar factores que inciden en su composición. Se gestionaron para la red 29 pozos a tres profundidades (acuífero profundo, intermedio y superficial) y se registró la temperatura, $\mathrm{CE}$ y pH en cuatro campañas entre 2016 y 2017. Los parámetros cuantificados a $<0,45 \mu \mathrm{m}$ fueron STD, iones mayoritarios, nitrato, elementos traza Cr-Mn-FeCo-Ni-Cu-Zn-Mo-Al-Cd-Pb-Hg-Li-Ba-B-As y los indicadores agronómicos \% Na, PSS, RAS, CSR y PS. Se observan diferencias espaciales, pero no temporales de la calidad de las aguas subterráneas termales, neutras a levemente alcalinas. La gran mayoría son aguas adecuadas para el riego de acuerdo a CE, RAS y CSR, y no existe riesgo por metales y metaloides; excepto por \% Na, PSS, SP, boro y litio (cítricos) de origen natural. El acuífero profundo ( $80-100 \mathrm{~m}$ ) posee aguas dulces de baja CE, bicarbonatadas y/o sulfatadas sódicas, pero el nivel de \% Na y PSS afecta el suelo; el intermedio (24-39 m) tiene aguas dulces y salobres, sulfatadas sódicas y \% Na, PSS y SP perjudicial. El acuífero superficial (17-21 m) contiene mayor salinidad (salobres), sódicas-cálcicas y se reparten en el límite entre sulfatada bicarbonatada o sulfatada clorurada, están bajo incumplimiento de normativas para cloruro, sulfato y nitrato. El aumento de iones disueltos en el acuífero superficial se atribuye a la disolución de yesiferas por aluvión en la zona, malas prácticas en procesos de riego-fertilización y la condición de aridez.
\end{abstract}

Palabras clave: acuífero, salinidad, normas de calidad, indicadores agronómicos

\begin{abstract}
Farmers from the Pica Oasis localities, foothills of northern Chile, take advantage of groundwater at different depths in the irrigation of citrus crops, tropical fruits and vegetables. In order to establish a monitoring network and provide recommendations to the irrigators, the physicochemical properties of these waters were characterized, their annual temporal behavior and their quality for irrigation was determined, as well as to interpret factors that affect the composition. 29 wells at three depths (deep, intermediate and superficial aquifer) were managed for the network and the temperature, $C E$ and $p H$ were recorded in four campaigns between 2016 and 2017. The parameters quantified at $<0.45 \mu \mathrm{m}$ were TDS, major ions, nitrate, trace elements $\mathrm{Cr}-\mathrm{Mn}-\mathrm{Fe}-\mathrm{Co}-\mathrm{Ni}-\mathrm{Cu}-\mathrm{Zn}$ Mo-Al-Cd-Pb-Hg-Li-Ba-B-As and the indicators $\% \mathrm{Na}$, SSP, SAR, RCS and PS. There are spatial variations, but not temporary, of the quality of the thermal groundwater, neutral to slightly alkaline. The vast majority are waters suitable for irrigation according to CE, RAS and CSR and there is no risk from metals and metalloids; except for\% Na, PSS, SP, boron and lithium (citrus). The deep aquifer (80-100 m) has fresh waters of low EC, bicarbonated and/or sulphated sodium, but the level of\% Na and PSS affects the soil; the intermediate $(24-39 \mathrm{~m})$ has fresh and brackish water, sulphated sodium and\% Na, PSS and harmful SP. The surface aquifer (17-21 m) contains higher salinity (brackish), sodium-calcium and is distributed in the boundary between sulfated bicarbonated or sulphated chloridated, are in breach of regulations for chloride, sulfate and nitrate. The increase of dissolved ions in the surface aquifer is attributed to the dissolution of sulphated by alluvium in the area, bad practices in irrigation-fertilization processes and the aridity condition.
\end{abstract}

Keywords: aquifer, salinity, quality standards, indicators

1 Universidad Arturo Prat. Facultad de Ciencias de la Salud. Centro de Investigación y Desarrollo en Recursos Hídricos, CIDERH. Iquique, Chile.

2 Departamento de Ciencias Geológicas, Universidad Católica del Norte. Antofagasta, Chile

3 Departamento de Ingeniería Química, Universidad Católica del Norte. Antofagasta, Chile.

4 Centro de Investigación Tecnológica del Agua en el Desierto CEITSAZA, Universidad Católica del Norte. Antofagasta, Chile.

* Autor por correspondencia: vherrera@unap.cl

Fecha de Recepción: 15 noviembre, 2017.

Fecha de Aceptación: 15 abril, 2018.

DOI: http://dx.doi.org/10.4067/S0718-34292018005000101. Publicado en línea: 17-julio-2018. 


\section{Introducción}

La composición natural de las aguas subterráneas es bastante compleja y depende de las condiciones hidrológicas e hidrogeológicas en la cuenca. Las características fisicoquímicas y biológicas propias de un cuerpo de agua permiten demostrar el estado, tanto en sentido temporal como espacial. La geoquímica interpreta los procesos físicos, geoquímicos y bioquímicos que controlan su composición química y que tienen incidencia o influencian la calidad. La actividad antrópica o natural introduce sustancias en el suelo superficial y en consecuencia se puede provocar su transporte hacia el acuífero (Custodio y Lamas, 2001; Belhady y Boutoutaou, 2017).

La calidad del agua obedece siempre a un objetivo de uso potencial que este recurso puede tener para la sociedad. Actualmente se utilizan diversos índices e indicadores agronómicos para evaluar la calidad de agua para riego, en los cuales se ponderan algunos parámetros fisicoquímicos medidos y calculados, tales como sólidos totales disueltos (STD), conductividad eléctrica (CE), bicarbonato $\left(\mathrm{HCO}_{3}^{-}\right)$, cloruro $(\mathrm{Cl}-)$, $\mathrm{pH}$, sodio $\left(\mathrm{Na}^{+}\right)$, relación de adsorción de sodio (RAS), porcentaje de sodio con base en cationes mayoritarios (\% Na), estimación del porcentaje de sodio soluble (PSS), el carbonato de sodio residual (CSR) y la salinidad potencial (SP) que relaciona los aniones cloruro y sulfato, entre otros (Ayers y Westcot, 1987; Arslan, 2016; Zaidi et al., 2016). En Chile, la legislación se encuentra establecida por el Instituto de Normalización en la Norma Chilena Oficial No1333 Of.197 (NCh1333) y en el ámbito internacional, principalmente por la Organización de las Naciones Unidas para la Alimentación y la Agricultura (FAO) (Instituto Nacional de Normalización, 1978; Ayers y Westcot,1987).

Para la región de Tarapacá, norte de Chile, el agua subterránea constituye el principal recurso hídrico permanente, característico de muchas zonas áridas del mundo. Las condiciones del perfil fisiográfico en altura han favorecido la acumulación de volúmenes de agua en los acuíferos de esta región cuya edad ha sido definida entre reciente y 10.000 años de antigüedad (Aravena, 1965; Magaritz et al., 1985; Grilli et al., 1999; DGA, 2012) y en consecuencia es considerado un recurso no renovable. Sin embargo, no se ha dado suficiente importancia al conocimiento de estos acuíferos, y más aún, todos presentan balance negativo, lo cual se materializa con un descenso continuo y paulatino de los niveles (Lictevout et al., 2013). El descenso, de manera general, se caracteriza también por el desmejoramiento de la calidad del agua (Belhady y Boutoutaou, 2017), aunque esta consideración no ha sido evaluada en la región. En el Oasis de Pica, dentro de la gran cuenca hidrográfica Pampa del Tamarugal, en las localidades Pica, Matilla y Quisma, sus aguas subterráneas fueron utilizadas para la producción agrícola de vino (siglo XVII) y desde finales del año 1920 hasta la actualidad (a pequeña y mediana escala) de cítricos (naranjo, limón de Pica, tangelo, pomelo), frutas tropicales (guayabo y mango) y verduras. Los agricultores con derechos de aprovechamiento de agua subterránea (DAA) a distintas profundidades, las benefician en un riego tradicional (por inundación), tecnificado y/o un riego mixto, desconociendo la calidad y sin una red de monitoreo. La información disponible de datos hidrogeoquímicos en vertientes naturales que afloran en el Oasis menciona aguas termales dulces, levemente alcalinas y del tipo bicarbonatadas sódicas y en general, aptas para riego (Digman y Galli, 1967; Salazar et al., 1999; Risacher et al., 2011; DGA, 2012). Con el objeto de establecer una red de monitoreo de salinidad y entregar recomendaciones a las asociaciones de regantes y agricultores, se evaluaron las propiedades fisicoquímicas de 29 muestras de aguas subterráneas y se determinó su calidad de acuerdo a indicadores agronómicos y estándares nacionales de riego y FAO, además de definir su comportamiento temporal anual e interpretar los factores que inciden en su composición.

\section{Materiales y métodos}

El Oasis de Pica se encuentra ubicado al piedemonte de la cordillera de los Andes (1.500 m.s.n.m.), en la subcuenca Quebrada de Quisma y subcuenca del mismo nombre de la zona hidrográfica Pampa del Tamarugal, cuyo modelo conceptual formula un sistema acuífero estratificado en multicapas (DGA, 2012). Se desarrolla un clima desértico normal caracterizado por una masa de aire estable y seca que provoca gran aridez, una humedad atmosférica baja que favorece una alta tasa de evaporación y escasa nubosidad. La precipitación promedia anual es de $14,3 \mathrm{~mm}$ en Pica y de aproximadamente $150 \mathrm{~mm}$ en Alto 
de Pica (3.500 m.s.n.m). Su régimen hidrológico incluye escurrimientos esporádicos superficiales y principalmente flujos de agua subterránea desde la cordillera de los Andes en dirección oeste. Prácticamente todo el escurrimiento se evapora o infiltra, sin embargo, existen flujos producto de afloramiento del acuífero en cinco vertientes, conocidas como "cochas" y socavones, con caudales regulares de 30 a $50 \mathrm{~L} / \mathrm{s}$ (Salazar et al., 1999; DGA, 2012). En Pica, Matilla y caseríos del valle de Quisma hay aproximadamente 3.000 habitantes y en el Censo Agropecuario del Instituto Nacional de Estadística (INE), Chile 1997, se estimó una superficie agrícola de 48.374 ha.
Con información recopilada desde la Dirección General de Aguas (DGA), Municipio de Pica y las Asociaciones de regantes y agricultores, y sus respectivas autorizaciones de ingreso a predios o “chacras", se logró evaluar las aguas subterráneas desde 29 pozos con DAA. Las campañas de terreno fueron desarrolladas en los meses de abril, julio y noviembre del año 2016, y enero del año 2017. Según la hondura del pozo se agruparon en acuífero profundo entre 80 y $100 \mathrm{~m}$, acuífero intermedio entre 24 y $39 \mathrm{~m}$, y acuífero superficial entre 17 y $21 \mathrm{~m}$ de profundidad. La Tabla 1 resume la ubicación geográfica (Datum WGS84) por localidad, la numeración de pozos y los intervalos

Tabla 1. Ubicación de sitios de la red de monitoreo, simbología e intervalos de valores obtenidos de temperatura, conductividad eléctrica y $\mathrm{pH}$ en las campañas de terreno.

\begin{tabular}{|c|c|c|c|c|c|c|c|}
\hline Acuífero & Localidad & $\mathbf{N}^{\circ}$ pozo & UTM_NORTE & UTM_ESTE & $\mathbf{T}\left({ }^{\circ} \mathbf{C}\right)$ & $\mathrm{CE}(\boldsymbol{\mu S} / \mathrm{cm})$ & pH (U) \\
\hline \multirow[t]{7}{*}{ Profundo } & Pica alto & 1 & 7735598,00 & 468121,00 & $32,8-33,6$ & $384-524$ & $8,40-8,65$ \\
\hline & Pica alto & 2 & 7734692,00 & 467269,00 & $30,9-33,2$ & $322-402$ & $7,70-8,22$ \\
\hline & Pica alto & 3 & 7734141,00 & 466219,00 & $27,8-29,9$ & $383-541$ & $7,51-7,91$ \\
\hline & Pica alto & 4 & 7734954,00 & 464231,00 & $28,1-29,5$ & $696-722$ & $7,14-7,87$ \\
\hline & Matilla & 5 & 7732774,00 & 460400,00 & $24,5-29,3$ & $1316-1653$ & $7,13-7,47$ \\
\hline & Quisma & 6 & 7731438,00 & 464404,00 & $29,5-30,3$ & $487-574$ & $8,55-8,75$ \\
\hline & Quisma & 7 & 7731406,00 & 464586,00 & $24,0-29,4$ & $682-910$ & $8,10-8,44$ \\
\hline \multirow[t]{14}{*}{ Intermedio } & Pica alto & 8 & 7734661,00 & 466144,00 & $26,7-27,3$ & $1237-155$ & $7,39-7,56$ \\
\hline & Pica alto & 9 & 7733612,00 & 464870,00 & $24,0-25,2$ & $2078-2327$ & $6,66-6,89$ \\
\hline & Pica alto & 10 & 7733353,00 & 464780,00 & $25,1-25,2$ & $1347-1465$ & $6,94-7,15$ \\
\hline & Pica bajo & 11 & 7733098,00 & 462872,00 & $26,4-27,0$ & $837-1034$ & $7,72-8,00$ \\
\hline & Pica bajo & 12 & 7732883,00 & 463079,00 & $21,0-23,4$ & $842-1023$ & $7,44-8,13$ \\
\hline & Pica bajo & 13 & 7732676,00 & 463644,00 & $22,6-23,4$ & $1614-1815$ & $7,19-7,55$ \\
\hline & Pica bajo & 14 & 7732395,00 & 463928,00 & $26,1-26,9$ & $1418-1420$ & $7,19-7,31$ \\
\hline & Pica bajo & 15 & 7732374,56 & 464395,76 & $20,7-21,3$ & $1004-1448$ & $6,62-7,06$ \\
\hline & Pica bajo & 16 & 7731964,00 & 463001,00 & $26,9-28,4$ & $847-911$ & $7,90-8,11$ \\
\hline & Matilla & 17 & 7731693,00 & 461651,00 & $22,3-24,7$ & $1203-1254$ & $6,50-8,24$ \\
\hline & Matilla & 18 & 7732546,00 & 460436,00 & $26,6-27,4$ & $1523-1927$ & $6,96-7,40$ \\
\hline & Quisma & 19 & 7731057,00 & 462378,00 & $29,8-33,5$ & $2615-2825$ & $7,44-7,77$ \\
\hline & Quisma & 20 & 7731063,00 & 463644,00 & $24,9-25,6$ & $1505-2373$ & $7,33-7,64$ \\
\hline & Quisma & 21 & 7731154,00 & 463680,00 & $24,9-26,8$ & $1622-2214$ & $7,41-7,54$ \\
\hline \multirow[t]{8}{*}{ Superficial } & Pica alto & 22 & 7733713,00 & 466014,00 & $23,5-24,3$ & $3542-3820$ & $7,14-7,40$ \\
\hline & Pica alto & 23 & 7736143,00 & 467790,00 & $31,7-31,9$ & $3203-3700$ & $7,22-7,83$ \\
\hline & Pica alto & 24 & 7735026,00 & 466472,00 & $24,7-24,9$ & $1087-1190$ & $7,62-7,89$ \\
\hline & Pica alto & 25 & 7734500,00 & 466809,00 & $24,2-24,7$ & $1265-1550$ & $6,89-7,15$ \\
\hline & Pica alto & 26 & 7734590,00 & 466187,00 & $25,1-27,2$ & $1982-2011$ & $7,23-7,51$ \\
\hline & Pica alto & 27 & 7734016,00 & 465577,00 & $22,9-23,4$ & $618-900$ & $6,84-7,18$ \\
\hline & Pica alto & 28 & 7733365,00 & 465668,00 & $29,4-35,1$ & $3656-3779$ & $6,83-7,20$ \\
\hline & Pica alto & 29 & 7737443,00 & 466212,00 & $24,8-25,9$ & $1340-2207$ & $8,15-8,38$ \\
\hline Flujo vertiente & Pica alto & Cocha & 7734755,00 & 466976,00 & $29,2-30,1$ & $320-333$ & $8,01-8,12$ \\
\hline
\end{tabular}


de la temperatura ( $\mathrm{T}), \mathrm{CE}$ y $\mathrm{pH}$, medidos en terreno con equipo multiparamétrico HANNA HI9828. También se incluye el sitio "cocha Resbaladero". Las muestras fueron colectadas en recipientes de polietileno y se fijaron con reactivos específicos según especie química a determinar. En el laboratorio químico del Centro de Investigación Tecnológica del Agua en el Desierto (CEITSAZA) de la Universidad Católica del Norte, Antofagasta, las muestras fueron filtradas a $0,45 \mu \mathrm{m}$ y se determinaron inmediatamente las concentraciones de bicarbonato y carbonato mediante titulación ácido-base con ácido clorhídrico estandarizado. Se determinaron los contenidos de STD por gravimetría a $105^{\circ} \mathrm{C}$, cloruro mediante el método de Morh, sulfato $\left(\mathrm{SO}_{4}{ }^{2-}\right)$ por gravimetría con cloruro de bario y nitrato $\left(\mathrm{N}-\mathrm{NO}_{3}{ }^{-}\right)$, por espectroscopia $\mathrm{UV}$ a 220 $\mathrm{nm}$ en espectrofotómetro Perkin Elmer Labda 25. Las determinaciones de las concentraciones de los metales mayoritarios $\mathrm{Na}, \mathrm{K}, \mathrm{Ca}$ y $\mathrm{Mg}$ y elementos traza Cr-Mn-Fe-Co-Ni-Cu-Zn-Mo$\mathrm{Al}-\mathrm{Cd}-\mathrm{Pb}-\mathrm{Hg}-\mathrm{Li}-\mathrm{Ba}-\mathrm{B}-\mathrm{As}$ fueron desarrolladas por espectrometría de emisión óptica de plasma de acoplamiento inductivo (ICP-OES) en equipo Perkin Elmer 7000 DV (APWA, 1992).

Para la validación, interpretación y presentación de los resultados obtenidos del análisis químico de los ocho iones mayoritarios $\left(\mathrm{Na}^{+}, \mathrm{K}^{+}, \mathrm{Ca}^{+2}, \mathrm{Mg}^{+2}, \mathrm{Cl}^{-}, \mathrm{SO}_{4}{ }^{-2}, \mathrm{HCO}_{3}{ }^{-} \mathrm{y} \mathrm{CO}_{3}{ }^{2-}\right) \mathrm{se}$ han considerado las muestras con un error de balance iónico menor que $\pm 10 \%$ (Custodio y Lamas, 2001). Se determinaron los indicadores agronómicos \% Na, PSS, RAS, CSR y SP (Instituto Nacional de Normalización, 1978; Ayers y Westcot, 1987; Arslan, 2016). Se revisaron los análisis de la red de monitoreo y se compararon los parámetros de estos con los estándares de NCh1333, y se clasificaron de acuerdo a lo establecido por la FAO.

\section{Resultados y discusión}

Las temperaturas (Tabla 1) corresponden a aguas termales, catastradas por Risacher et al., (2011). Las más elevadas se registraron en el acuífero profundo, 29,9 $\pm 2,4{ }^{\circ} \mathrm{C}$, similar al afloramiento natural en la cocha. Los acuíferos intermedio y superficial poseen temperaturas menores y parecidas $25,2 \pm 2,4$ y $25,5 \pm 2,7{ }^{\circ} \mathrm{C}$, respectivamente, probablemente por el agua fría de retorno a menos profundidad del exceso de riego. Las mediciones de $\mathrm{pH}$ las clasifican como neutras a levemente alcalinas y exhibe un valor levemente mayor el acuífero profundo $(8,01 \pm 0,52)$. La NCh1333 establece un amplio intervalo entre 5,5 y 9,0 unidades de $\mathrm{pH}$ y las aguas naturales conservan el $\mathrm{pH}$ entre 6,5 y 8,5 (Custodio y Lamas, 2001).

La salinidad, expresada como STD y CE, es herramienta importante en aguas de riego, presentando el Oasis un intervalo amplio (188 a $3082 \mathrm{mg} / \mathrm{L}$ y 320 a $3826 \mu \mathrm{S} / \mathrm{cm})$. Se encontraron aguas menos salinas en el acuífero profundo (670 $\pm 360 \mu \mathrm{S} / \mathrm{cm}$ ); duplicando su CE en el acuífero intermedio $(1496 \pm 497 \mu \mathrm{S} / \mathrm{cm})$ y triplicando su valor en el acuífero superficial $(2252 \pm 1156 \mu \mathrm{S} /$ $\mathrm{cm})$. La NCh1333 establece que no se observarán efectos perjudiciales en cultivos si STD es inferior a $500 \mathrm{mg} / \mathrm{L}$ y CE inferior a $750 \mu \mathrm{S} / \mathrm{cm}$; aguas que pueden tener efectos perjudiciales en cultivos sensibles si sus STD se encuentran entre 500 y $1000 \mathrm{mg} / \mathrm{L}$ y sus CE entre 750 y 1500 $\mu \mathrm{S} / \mathrm{cm}$, y aquellas aguas que sobrepasan estas concentraciones (Tabla 2) pueden tener efectos adversos en muchos cultivos y necesitan métodos de manejo, como es el caso de chacras en Pica alto con abastecimiento desde aguas salobres menos profundas (acuífero intermedio y superficial).

La Figura 1 muestra el registro temporal de los STD para los pozos, demostrándose un leve aumento en época seca y calurosa, y corrobora que las aguas menos profundas adquieren los valores más altos. De acuerdo a estudios previos de las vertientes termales y algunos pozos construidos en el Oasis, coincidieron en los intervalos de valores de CE, STD y pH (Salazar et al.,1999; DGA, 2012). La clasificación geoquímica por STD estableció que, del total de muestras, $57 \%$ corresponden a aguas dulces y $43 \%$ a salobres (Drever, 1997). En el acuífero profundo, el 96\% de muestras han sido clasificadas como dulces, con escaso contenido iónico en comparación con otras aguas subterráneas de la región (Lictevout et al., 2013) y zonas de aridez extrema (Belhady y Boutoutaou, 2017). En el acuífero intermedio, solamente el $55 \%$ de las muestras son dulces, pero en el acuífero superficial, el $74 \%$ de las muestras presentaron aguas salobres (Tabla 2). Los riegos continuados con aguas salobres pueden traer como consecuencia la salinización de los suelos, reduciendo la disponibilidad de agua para la planta (Arslan, 2016; Zaidi et al., 2016). El aumento de 
Tabla 2. Idoneidad de las muestras de aguas subterráneas de los acuíferos profundo, intermedio y superficial, para el riego en el Oasis de Pica, con base en indicadores de calidad.

\begin{tabular}{|c|c|c|c|c|c|c|c|c|}
\hline \multirow[b]{3}{*}{ Indicadores } & \multirow[b]{3}{*}{ Categorías } & \multirow[b]{3}{*}{ Intervalos } & \multicolumn{6}{|c|}{ Muestras } \\
\hline & & & \multicolumn{2}{|c|}{ Profundo } & \multicolumn{2}{|c|}{ Intermedio } & \multicolumn{2}{|c|}{ Superficial } \\
\hline & & & $\mathrm{N}^{\circ}$ & $\%$ & $\mathrm{~N}^{\circ}$ & $\%$ & $\mathrm{~N}^{\circ}$ & $\%$ \\
\hline \multirow[t]{3}{*}{$\mathrm{CE}(\mu \mathrm{S} / \mathrm{cm})$} & Permisible & $<1500$ & 27 & 96 & 36 & 68 & 12 & 39 \\
\hline & Dudosa & $1500-3000$ & 1 & 4 & 17 & 32 & 8 & 26 \\
\hline & Peligrosa & $>3000$ & 0 & 0 & 0 & 0 & 11 & 35 \\
\hline \multirow[t]{2}{*}{ STD (mg/L) } & Dulce & $<1000$ & 27 & 96 & 29 & 57 & 8 & 26 \\
\hline & Salobre & $1000-10000$ & 1 & 4 & 24 & 43 & 23 & 74 \\
\hline \multirow[t]{5}{*}{$\% \mathrm{Na}(\%)$} & Excelente & $<20$ & 0 & 0 & 0 & 0 & 0 & 0 \\
\hline & Buena & $20-40$ & 2 & 7 & 0 & 0 & 2 & 6 \\
\hline & Permisible & $40-60$ & 4 & 14 & 23 & 42 & 26 & 84 \\
\hline & Dudosa & $60-80$ & 16 & 57 & 29 & 56 & 3 & 10 \\
\hline & Inadecuada & $>80$ & 6 & 21 & 0 & 0 & 0 & 0 \\
\hline \multirow[t]{2}{*}{ SSP $(\%)$} & Buena & $<50$ & 2 & 7 & 5 & 9 & 12 & 37 \\
\hline & Mala & $>50$ & 26 & 93 & 50 & 91 & 19 & 63 \\
\hline \multirow[t]{4}{*}{ SAR (meq/L) } & Excelente & $<10$ & 24 & 86 & 56 & 91 & 31 & 100 \\
\hline & Buena & $10-18,0$ & 4 & 14 & 7 & 7 & 0 & 0 \\
\hline & Dudosa & $18-26$ & 0 & 0 & 0 & 0 & 0 & 0 \\
\hline & Inadecuada & $>26$ & 0 & 0 & 0 & 0 & 0 & 0 \\
\hline \multirow[t]{3}{*}{$\operatorname{RSC}(\mathrm{meq} / \mathrm{L})$} & Buena & $<1,25$ & 28 & 100 & 56 & 100 & 31 & 100 \\
\hline & Media & $1,25-2,5$ & 0 & 0 & 0 & 0 & 0 & 0 \\
\hline & Mala & $>2,5$ & 0 & 0 & 0 & 0 & 0 & 0 \\
\hline \multirow[t]{3}{*}{$\mathrm{SP}(\mathrm{meq} / \mathrm{L})$} & Buena & $<3$ & 16 & 57 & 0 & 0 & 4 & 13 \\
\hline & Media & $3,0-15$ & 12 & 43 & 53 & 100 & 15 & 48 \\
\hline & Inadecuada & $>15$ & 0 & 0 & 0 & 0 & 12 & 39 \\
\hline
\end{tabular}

CE: conductividad eléctrica; STD: sólidos totales disueltos; \% Na sodio porcentual; SSP: porcentaje de sodio soluble; SAR: relación de adsorción de sodio; RSC: carbonato de sodio residual y SP: salinidad potencial. meq/L: unidad de medición en miliequivalentes del ion por litro de muestra.

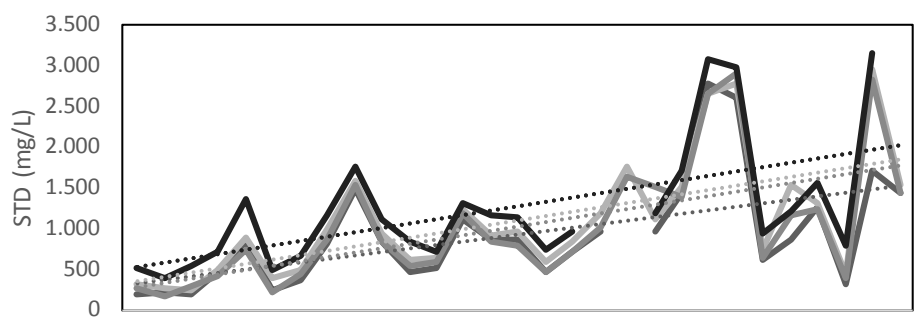

12345678991011121314151617181920212223242526272829 sitios

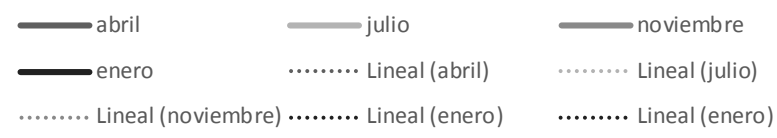

Figura 1. Registro temporal (2016 - 2017) de STD para los sitios de la red de monitoreo 
la salinidad, además de la evaporación, se atribuye a una contaminación natural producto del arrastre con gran fuerza de una mezcla de agua, rocas y sedimentos, por efecto de las lluvias estivales intensas en el Altiplano, y caudales intermitentes en la quebrada de Quisma, que provocan recargas a través de un pequeño abanico aluvial (Houston, 2001).

La salinidad también se relaciona con el contenido y predominio de los iones mayoritarios en disolución. Los que adquieren las mayores concentraciones son sodio y calcio, sobrepasando en todas las muestras el $90 \%$ del contenido catiónico. Los iones potasio y magnesio alcanzan muy bajas concentraciones y similares. El anión sulfato predomina en la mayoría de las muestras: solamente 2 muestras del acuífero profundo poseen anión bicarbonato como mayoritario. El pH de las aguas inferior a 8,3 limita la concentración de carbonatos y la proporción de cloruro aumenta en aguas salobres. La clasificación geoquímica (Custodio y Lamas, 2001) por iones dominantes establece un quimismo complejo: en el acuífero profundo, las aguas en el área de cultivos intensos en Pica alto (2 y 3 ) son del tipo bicarbonatadas sulfatadas sódicas, $\mathrm{Na}^{+} / \mathrm{HCO}_{3}{ }^{-}>\mathrm{SO}_{4}{ }^{-2}$, similar a lo observado para las aguas de la vertiente termal cocha; el bicarbonato se relaciona con presencia de materia orgánica, pero en suelo desnudo (1), el quimismo se invierte ya que las aguas son del tipo sulfatadas bicarbonatadas sódicas $\mathrm{Na}^{+} / \mathrm{SO}_{4}{ }^{-2}$ $>\mathrm{HCO}_{3}{ }^{-}$. En el sector de Matilla (4 y 5) las aguas son sulfatadas bicarbonatadas cloruradas $\mathrm{Na}^{+} /$ $\mathrm{SO}_{4}^{-2}>\mathrm{HCO}_{3}>\mathrm{Cl}^{-}$y las del sector de Quisma son sulfatadas cloruradas sódicas $\mathrm{Na}^{+} / \mathrm{SO}_{4}{ }^{-2}>\mathrm{Cl}^{-}$. En el acuífero intermedio, en su mayoría son del tipo sulfatadas sódicas, $\mathrm{Na}^{+} / \mathrm{SO}_{4}^{-2}$, y en el sector Pica bajo son sulfatadas cloruradas sódicas, $\mathrm{Na}^{+} / \mathrm{SO}_{4}{ }^{-}$ ${ }^{2}>\mathrm{Cl}^{-}$. En el acuífero superficial son sulfatadas sódicas, $\mathrm{Na}^{+} / \mathrm{SO}_{4}^{-2}$, y en aguas con mayor $\mathrm{CE}$, resultan importantes el ion cloruro y el calcio. Existe un quimismo desde aguas bicarbonatadas a sulfatadas y posteriormente cloruradas por las mezclas de distintas fuentes de agua, disolución de sulfato y cloruro de sodio que hay en la cuenca y también por efecto de evaporación. Cloruro, ion conservativo en aguas, es un anión esencial para la vida, pero a concentraciones elevadas en aguas de riego implica toxicidad para muchas plantas, se presenta entre 60 y $280 \mathrm{mg} / \mathrm{L}$. La NCh1333 establece un límite máximo permitido de $200 \mathrm{mg} / \mathrm{L}$. El anión sulfato, que posee sales moderadamente solubles a muy solubles, se encuentra en un amplio intervalo y variable $(371 \pm 326 \mathrm{mg} / \mathrm{L})$, y varios pozos en el acuífero superficial están bajo incumplimiento de las NCh1333, que no deben superar $250 \mathrm{mg} / \mathrm{L}$.

Los resultados de los indicadores agronómicos, según su categoría e intervalo, son mostrados en la Tabla 2. La NCh1333 utiliza el indicador \% Na, lo que está directamente relacionado con contenidos de magnesio y calcio, y con un máximo permitido de $35 \%$. En el Oasis la mayoría de las muestras de agua sobrepasaron este valor estándar, acentuando el riesgo en las aguas más profundas y siendo perjudicial para las plantas al reducir la permeabilidad en suelos. Los valores sobre el 50\% del PSS evidencian aguas subterráneas de mala calidad en relación con sodio adverso para el suelo, a mayor profundidad en la cuenca, aunque estas sean de baja salinidad. Sin embargo, los valores obtenidos para el RAS y CSR indican que las aguas subterráneas son adecuadas para el riego, incluso clasifican de excelente RAS todas aquellas muestras salobres del acuífero menos profundo. Los valores elevados de SP (87\%), que relaciona cloruros y sulfatos, limitan la calidad del acuífero superficial a cultivos moderadamente tolerantes a estas sales.

El nivel de nitrato en las aguas subterráneas del Oasis mostró diferencias importantes (Figura 2). El acuífero profundo presentó concentraciones bajas, pero en el acuífero intermedio hay un incremento de tres a cinco veces y en el acuífero superficial podemos afirmar que hay contaminación. La NCh1333 no lo incluye como indicador, pero la FAO sugiere una concentración no superior a $30 \mathrm{mg} / \mathrm{L}$, dados los efectos adversos en una variedad de plantas (Ayers y Westcot, 1987; Arslan, 2016). Las causas probables del aumento del nutriente son las actividades agrícolas intensivas y el sistema de drenaje en las prácticas del riego por el arrastre del nitrato no aprovechado por debajo de la zona radicular, produciendo la contaminación de las aguas en esta zona del Oasis. No se encontró relación entre la salinidad y las concentraciones de nitrato en las aguas.

En el Oasis, ninguno de los metales esenciales (Cr-Mn-Fe-Co-Ni-Cu-Zn-Mo-Al) ni los metales tóxicos $(\mathrm{Cd}-\mathrm{Hg}-\mathrm{Pb})$ sobrepasanlasconcentraciones máximas admisibles por NCh1333 y no significan 


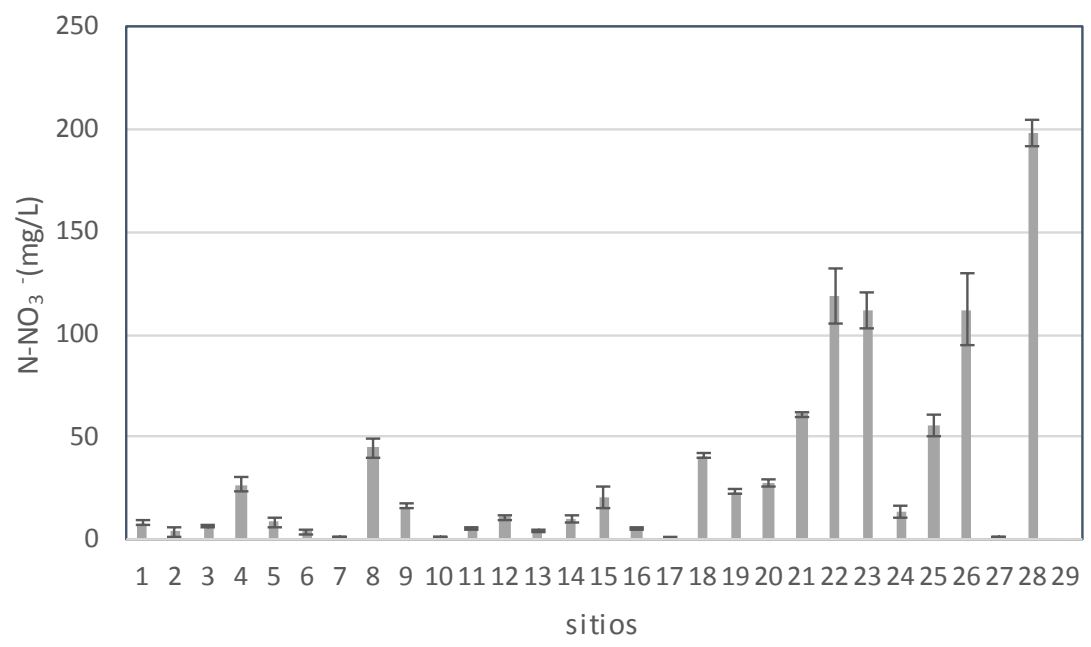

Figura 2. Concentración de nitrato en aguas subterráneas para los sitios de la red de monitoreo (riesgo > 30 mg/L, según FAO).

riesgo (Tabla 3). Sin embargo, las condiciones de aridez, geotermalismo, volcanismo activo y actividades de la minería en el norte grande de Chile han sido argumentos para interpretar concentraciones elevadas de elementos traza en las aguas subterráneas, tales como silicio, litio, boro y arsénico (Aravena, 1965; Risacher et al., 2003 y 2011; Lictevout et al., 2013). A diferencia de lo observado en una variedad de cuerpos de agua en la región de Tarapacá (Lictevout et al., 2013), la concentración de arsénico en los acuíferos de Pica $(0,02 \pm 0,03 \mathrm{mg} / \mathrm{l})$ no sobrepasa la NCh1333 $(0,10 \mathrm{mg} / \mathrm{L})$.

Boro en pequeñas cantidades es esencial para el crecimiento de las plantas, sin embargo, en concentraciones excesivas, tanto en suelos como en el agua de riego, puede tener elevada toxicidad para ciertos cultivos sensibles (Reid, 2010). La Figura 3 presenta la distribución de boro en las aguas subterráneas y la mayoría de las muestras acusan una concentración superior a aquellas sugeridas por la NCh1333 que es $0,75 \mathrm{mg} / \mathrm{L}$, aunque la FAO considera un contenido normal entre 0 y $2 \mathrm{mg} / \mathrm{L}$. La Figura 4 muestra que existe una relación directa entre este parámetro y STD, lo que implica que el aumento de boro en las aguas menos profundas sea consecuencia de procesos de lixiviación del terreno por el cual circula y de evaporación. Boro de forma natural en el norte de Chile procede de la alteración de rocas ígneas, gases volcánicos y terrenos evaporíticos (Risacher et al., 2003). Siete parámetros son medidos por NCh1333 y FAO, siendo más restrictiva la norma chilena para boro, cloruro, sulfato y arsénico.

Las concentraciones de litio en las aguas subterráneas del Oasis (Figura 5) son importantes, ya que comparte propiedades con sodio y otros metales alcalinos, se le atribuye origen termal (Risacher et al., 2011) y por su efecto en los cultivos cítricos, principal actividad económica. La NCh1333 considera una concentración máxima admisible de $2,5 \mathrm{mg} / \mathrm{L}$, valor que no se alcanza en las aguas en estudio, pero la misma norma tiene restricción a $0,075 \mathrm{mg} / \mathrm{L}$ para cultivos cítricos y en este caso, la mayoría de las muestras de agua supera tal concentración. No existe relación con STD y Shahzad et al., (2016) mencionan que la toxicidad del litio es incierta en las plantas y que probablemente interrumpe procesos fisiológicos y altera el metabolismo. En Pica hay un déficit de aproximadamente 1.200 a $1.500 \mathrm{~mm}$ por año entre las precipitaciones y la evaporación (DGA, 2012), y de acuerdo a los resultados, la sobreexplotación del recurso hídrico y el calentamiento global influyen en su calidad y aumentará la concentración de los metales alcalinos, alcalinos térreos y aquellos aniones asociados (boratos, sulfatos y cloruros). 
Tabla 3. Concentración máxima admisible (NCh1333) para elemento traza, el promedio, desviación estándar, límite de detección del método y valor máximo para la serie de datos.

\begin{tabular}{llrrrrr}
\hline Elemento/Parámetro & $\begin{array}{r}\text { NCh1333 } \\
(\mathrm{mg} / \mathrm{L})\end{array}$ & $\begin{array}{r}\text { Promedio } \\
(\mathrm{mg} / \mathrm{L})\end{array}$ & Desviación & LD $(\mathrm{mg} / \mathrm{L})$ & $\begin{array}{r}\text { Max } \\
(\mathrm{mg} / \mathrm{L})\end{array}$ \\
\hline $\mathrm{Cr}$ & Cromo & 0,10 & $7 \times 10^{-5}$ & 0,0 & $7 \times 10^{-5}$ & $7 \times 10^{-5}$ \\
$\mathrm{Mn}$ & Manganeso & 0,20 & 0,154 & 0,553 & $1 \times 10^{-4}$ & 0,95 \\
$\mathrm{Fe}$ & Hierro & 5,0 & 0,073 & 0,220 & $5 \times 10^{-4}$ & 1,60 \\
$\mathrm{Co}$ & Cobalto & 0,05 & $4 \times 10^{-4}$ & $3,6 \times 10^{-4}$ & $5 \times 10^{-4}$ & $2 \times 10^{-4}$ \\
$\mathrm{Ni}$ & Níquel & 0,2 & 0,060 & 0,163 & $4 \times 10^{-4}$ & 0,50 \\
$\mathrm{Cu}$ & Cobre & 0,2 & $1,3 \times 10^{-4}$ & $1,3 \times 10^{-4}$ & $1,3 \times 10^{-4}$ & $1,3 \times 10^{-4}$ \\
$\mathrm{Zn}$ & Cinc & 2,0 & 0,041 & 0,147 & $5 \times 10^{-4}$ & 0,80 \\
$\mathrm{Mo}$ & Molibdeno & 0,01 & $3 \times 10^{-4}$ & $1,7 \times 10^{-4}$ & $5 \times 10^{-4}$ & $5 \times 10^{-4}$ \\
$\mathrm{Al}$ & Aluminio & 5,0 & $3 \times 10^{-4}$ & 0,0 & $1 \times 10^{-4}$ & $3,3 \times 10^{-4}$ \\
$\mathrm{Cd}$ & Cadmio & 0,01 & $1 \times 10^{-5}$ & 0,0 & $1 \times 10^{-4}$ & $3,3 \times 10^{-4}$ \\
$\mathrm{~Pb}$ & Plomo & 5,0 & $1,7 \times 10^{-4}$ & $1,6 \times 10^{-4}$ & $5 \times 10^{-5}$ & $3,3 \times 10^{-4}$ \\
$\mathrm{Ba}$ & Bario & 4,0 & 0,026 & 0,03 & $3 \times 10^{-4}$ & 0,096 \\
$\mathrm{As}$ & Arsénico & 0,10 & 0,019 & 0,03 & $2 \times 10^{-3}$ & $1 \times 10^{-3}$ \\
$\mathrm{Hg}$ & Mercurio & 0,01 & $1 \times 10^{-3}$ & 0,0 & $1 \times 10^{-3}$ \\
\hline
\end{tabular}

LD: Límite de detección; Max: valor máximo de la serie de datos

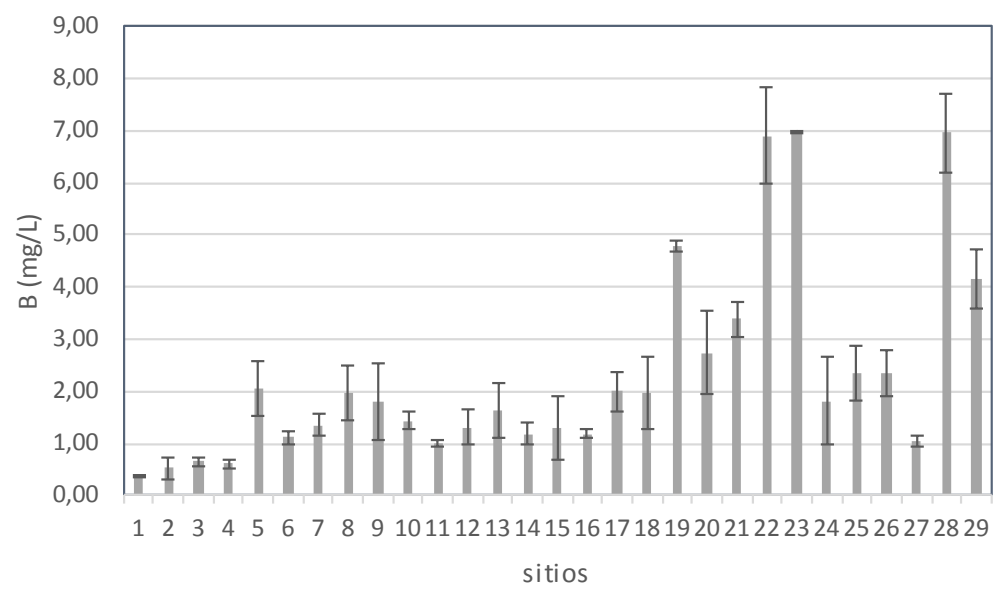

Figura 3. Concentración de boro en aguas subterráneas para los sitios de la red de monitoreo y concentración máxima admisible NCh1333 (0,75 mg/L) 


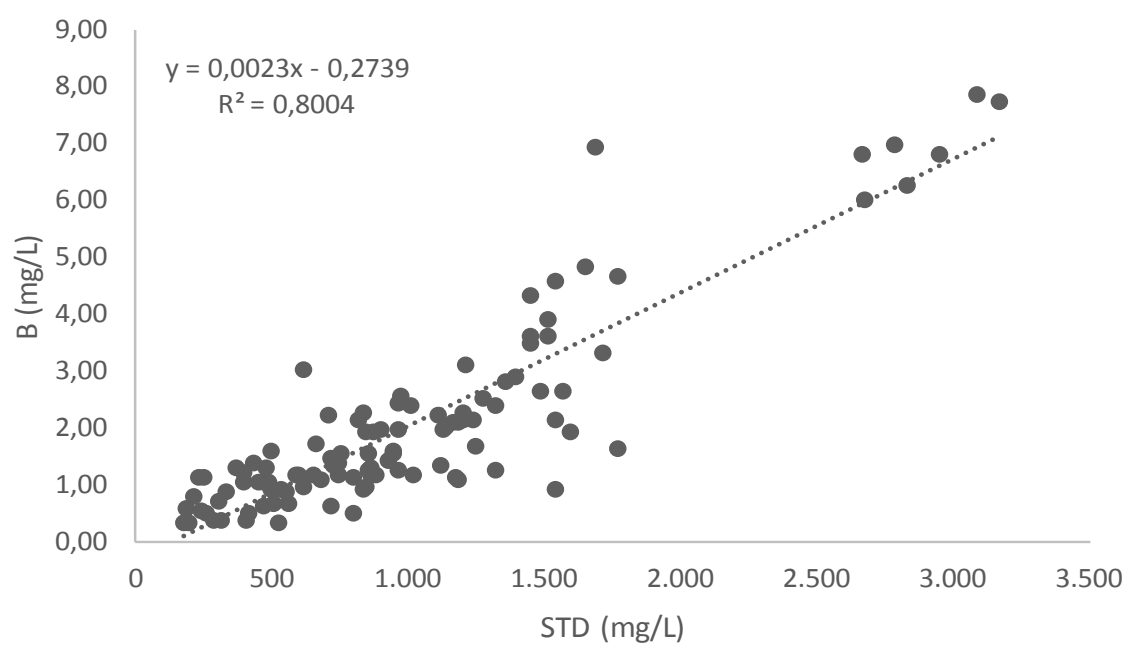

Figura 4. Relación entre la concentración de boro y STD en aguas subterráneas para los sitios de la red de monitoreo.

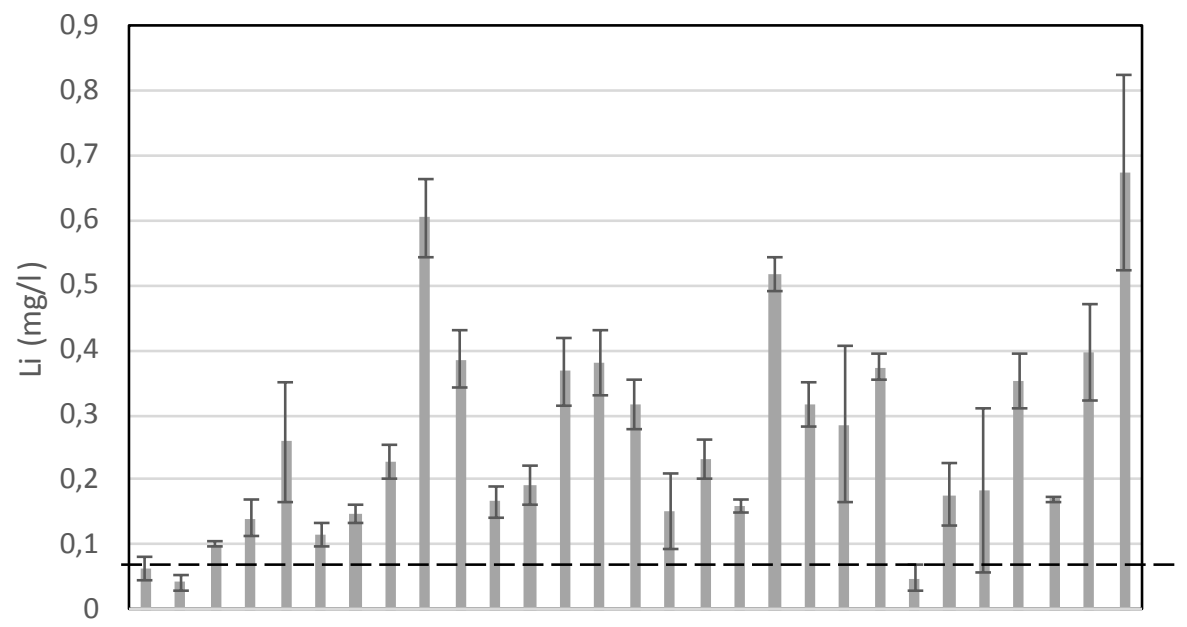

12234456678891011121314151617181920212223242526272829

sitios

Figura 5. Concentración de litio en aguas subterráneas para los sitios de la red de monitoreo y concentración máxima admisible NCh1333 para cítricos $(0,075 \mathrm{mg} / \mathrm{L})$ 


\section{Conclusión}

Las aguas subterráneas termales, neutras y levemente alcalinas del Oasis de Pica son su mayoría adecuadas para el riego según valores de CE, RAS y CSR, y no hay riesgo por metales y metaloides traza, excepto por boro y litio (cítricos) de acuerdo a estándares de NCh1333 y FAO. Pero, según su profundidad en la cuenca hidrográfica, las aguas presentan diferencias en su composición e idoneidad para el riego. El acuífero profundo posee aguas dulces de menor contenido iónico y con predominio en sodio, sulfato y bicarbonato, aunque por valores de \% Na y PSS provocan efectos adversos en los suelos. El acuífero intermedio además tiene aguas salobres y con predominio en sodio y sulfato, posee valores no aptos de \% Na y SSP y medios de SP. El acuífero superficial presenta aguas salobres, más salinizadas, pero con valores permitidos de \% Na y PSS, aunque están bajo incumplimiento de normas nacionales para sulfato y cloruro, y de acuerdo a la FAO sobrepasan los máximos permitidos para SP y nitrato. Su composición química por iones mayoritarios establece aguas sódicas-cálcicas y se reparten en el límite entre sulfatada y bicarbonatada; las más diluidas varían de un polo bicarbonatado a sulfatado, mientras que aquellas enriquecidas con iones en disolución van desde un sistema sulfatado hasta uno clorurado. Inciden en la calidad del agua del acuífero superficial la evaporación, los eventos de disolución de yesiferas y, probablemente, por sobreexplotación del recurso y malas prácticas en riego-fertilización.

\section{Agradecimientos}

Agradecemos al Gobierno Regional de Tarapacá, que ha financiado el proyecto FIC-R 2015 BIP 304349260; a la Universidad Arturo Prat del estado de Chile, a la Universidad Católica del Norte, a la Ilustre Municipalidad de Pica y a las Asociaciones de regantes y agricultores del Oasis de Pica.

\section{Literatura citada}

\section{APHA-AWWA-WPCF}

1992. Métodos normalizados para el análisis de aguas potables y residuales. Ediciones Díaz de Santos, S.A. Madrid, España. 1830p.

Aravena, R.

1995. Isotope Hidrology and Geochemistry of Northern Chile Groundwaters. Bulletin de l'Institut Français d'Études Andines, 24 (3): 495-503.

Arslan, S.

2016. Assessment of groundwater and soil quality for agricultural purposes in Kopruoren basin, Kutahya. Turkey. Journal of African Earth Sciences, 131: 1-13.

Ayers, R.; Westcot, S.

1987. La calidad del agua y su uso en la agricultura. Estudio FAO, Riegos y Drenajes Nº 29 Rev.1. Roma, Italia. 174p.

Belhady, R.; Boutoutaou, D.

2017. Characterization of groundwater in arid zones (Case of Ouargla basin). Energy Procedia, 119: 556 - 564.

Custodio, E.; Llamas, M. R.

2001. Hidrología Subterránea. Tomo I, Sección 10 Hidrogeoquímica. Omega, S.A. Barcelona, España. 1157p.

Dirección General de Agua, Ministerio de Obras Públicas, DGA-MOP.

2012. Levantamiento de información hidrogeológica para la modelación del acuífero de Pica, región de Tarapacá. Santiago, Chile. 193p.
Digmam, J; Galli, C.

1965. Geology and Ground-Water Resources of the Pica Area Tarapaca Province, Chile. Geological Survey Bulletin 1189, Washington, US. 129 p.

Drever, J.

1997. Geochemistry of natural waters: The surface and groundwater environments. Prentice-hall, Upper Saddle River. US. 436 p.

Grilli, A.; Aguirre, E.; Durán, M.; Townsend, F.; González, A. 1999. Origen de las aguas subterráneas del sector Pica-Salar del Huasco, provincia de Iquique, I región de Tarapacá. XIII Congreso de Ingeniería Sanitaria y Ambiental AIDIS, Antofagasta, Chile.18 p.

Houston, J.

2001. La precipitación torrencial del año 2000 en quebrada Chacarilla y el cálculo de recarga al acuífero Pampa Tamarugal, norte de Chile. Rev. Geol. Chile, 28(2): 163-177

Lictevout, E.; Maass C.; Córdoba, D.; Herrera, V.; Payano, R. 2013. Diagnóstico y sistematización de la información de los recursos hídricos de la Región de Tarapacá, Chile. Centro de Investigación en Recursos Hídricos (CIDERH), Universidad Arturo Prat. Iquique, Chile. 223p.

Magaritz, M.; Suzuki, O.; Peña, H.

1985. Isotopic and chemical study of the water resources in the Iquique Province. Dirección General de Aguas, Ministerio de Obras Públicas. Santiago, Chile. 62p. 
Instituto Nacional de Normalización.

1978. NCh 1.333.Of78 Modificada 1987. Requisitos de Calidad del Agua para Diferentes Usos. Santiago, Chile. 15p.

Salazar, C.; Roja, L.; Pollastri, A.

1999. Evaluación de recursos hídricos en el sector de Pica, hoya de la Pampa del Tamarugal. I región, Chile. Dirección General de Aguas. Ministerio de Obras Públicas. Chile. 16p.

Shahzad, B.; Tanveer, M.; Hassan, W.; Shah, A.; Anjum, Sh.; Cheema, S.; Ali, I.

2016. Lithium toxicity in plants: Reasons, mechanisms and remediation possibilities. A review. Plant Physiology and Biochemistry, 107: 104-115.
Reid, R.

2010. Can we really increase yields by making crop plants tolerant to boron toxicity? Plant Science, 178: 9-11.

Risacher, F.; Alonso, H.; Salazar, C.

2003. The origin of brines and salts in Chilean salars. A hydrochemical review. Earth Sciences, 63: 249 -293.

Risacher, F.; Fritz, B.; Hauser, A.

2011. Origin of components in Chilean thermal waters. Journal of South American Earth Sciences, 31: 153-170.

Zaidi F.; Mogren S.; Mukhopadhyay, M.; Ibrahim, E.

2016. Evaluation of groundwater chemistry and its impact on drinking and irrigation water quality in the eastern part of the Central Arabian graben and trough system, Saudi Arabia. Journal of African Earth Sciences, 120: 208-219. 